\title{
Semaphorin 4D activates the MAPK pathway downstream of plexin-B1
}

\author{
Jennifer AURANDT* ${ }^{*}$, Weiquan $\mathrm{LI}^{*}$ and Kun-Liang GUAN ${ }^{*} \dagger t^{1}$ \\ *Life Sciences Institute, University of Michigan, Ann Arbor, MI 48109, U.S.A., †Department of Biological Chemistry, University of Michigan, Ann Arbor, MI 48109, U.S.A., \\ and $\ddagger$ Institute of Gerontology, University of Michigan, Ann Arbor, MI 48109, U.S.A.
}

Semaphorins are a large family of transmembrane and secreted proteins that signal primarily through the receptor plexin. Semaphorins have been characterized in the nervous system as axon guidance cues; however, they have also been shown to control development of other cellular systems such as the vasculature and lungs. As the role of semaphorins outside of the nervous system has broadened, so has elucidation of the intracellular signalling pathways they initiate. Previously, we and others have shown that plexin-B1 activates RhoA through the binding and activation of RhoGEF (guanine nucleotide-exchange factor)/LARG (leukaemia-associated RhoGEF) in response to semaphorin 4D stimulation. In the present study, we show that semaphorin 4D activates the MAPK (mitogen-activated protein kinase) pathway. We have found that the mechanism of activation requires the C-terminus of plexin-B1 and the activation of RhoA.

Key words: axon guidance, HT-22 cell, mitogen-activated protein kinase (MAPK), plexin, semaphorin, Rho GTPase.

\section{INTRODUCTION}

Semaphorins are a family of soluble and membrane-associated proteins that were originally characterized in the nervous system and play a critical role in axon guidance. Outside of the nervous system, semaphorins mediate the immune response [1], tumour growth [2] and development of the vasculature [3,4], lung [5] and heart [6,7]. More than 20 semaphorins have been identified to date and are grouped into eight classes. Invertebrate semaphorins are based in Class 1 and 2; viral semaphorins in Group V, and Classes 3-7 comprise the vertebrate semaphorins. The physiological functions of semaphorins are mediated through a family of transmembrane receptors called plexins. Nine plexins can be divided into four subfamilies: plexin-A1-A4, plexin-B1-B3, plexin-C1 and plexin-D1. Secreted semaphorins, such as Sema3A (semaphorin $3 \mathrm{~A}$ ), require a co-receptor, such as neuropilin, to bind and then initiate intracellular signalling through plexin. Alternatively, a transmembrane member of the semaphorin family, Sema4D, mediates signalling through direct binding to the transmembrane receptor plexin-B1.

Binding of semaphorin to plexin initiates a signalling cascade, which varies depending on the semaphorin/plexin family pair. Most recently, Oinuma et al. [8] found that plexin-B1 and plexinA1 contain GAP (GTPase-activating protein) activity toward RRas. R-Ras plays a known role in regulating cellular adhesion, and plexin inactivation of R-Ras may signal to limit integrin-mediated cellular adhesion [9]. Furthermore, Oinuma et al. [8] showed that plexin-B1 requires direct binding of Rnd1, a Rho GTPase family member, for full GAP activity. This discovery defines a novel class of receptors that contain intrinsic GAP activity. Furthermore, Rho family GTPases are regulated by semaphorin/plexin interaction. We [10] and others [11] have shown that activated Rac directly binds to a CRIB (Cdc42/Rac interactive binding) domain present in plexin-B family members. Both biochemical and genetic data suggest that plexin-B1 may compete with PAK (p21-activated kinase) for Rac binding; therefore plexin-B1 sequesters Rac and inhibits PAK activation. Rac may also play a role in the ligandbinding function of plexin-B1 [12]. Rho has also been implicated through Drosophila genetics to directly bind to plexin-B. This interaction was essential for correct axon guidance in plexin-Bexpressing ISNb neurons [13]. Interestingly, mammalian plexinB1 does not bind Rho. However, we [25] and others [14,26,27] have found that LARG [leukaemia-associated RhoGEF (guanine nucleotide-exchange factor)] and PRG (PDZ-RhoGEF) are Rhospecific GEFs that bind to the C-terminus of plexin-B1 and mediate RhoA activation in response to Sema4D stimulation. Binding of LARG or PRG and consequent activation of RhoA were shown to cause Sema4D-stimulated growth-cone collapse in hippocampal cells [14].

Activation of the MAPK (mitogen-activated protein kinase) family has been observed in response to many axon guidance molecules such as netrin, Sema7A and Sema3A [15,16]. In the developing nervous system, ERK (extracellular-signal-regulated kinase) activation has been shown to contribute to the axon turning response, protein synthesis in axons and neurite outgrowth [1619]. However, the mechanism of ERK activation downstream of Sema3A is unknown. Therefore, to determine whether Sema4D treatment activates ERK and by what mechanism, we stimulated a hippocampal cell line, HT-22, with purified Sema4D and found that ERK phosphorylation was increased. We found that activation of ERK occurred through RhoA activation. Our results suggest a mechanism by which ERK is activated downstream of Sema4D/plexin-B1 signalling.

\section{MATERIALS AND METHODS}

\section{Plasmids and antibodies}

Antibodies against phospho-ERK and total ERK were from Biosource, the antibody against HA (haemagglutinin) was from

Abbreviations used: AP, alkaline phosphatase; DMEM, Dulbecco's modified Eagle's medium; DTT, dithiothreitol; ERK, extracellular-signal-regulated kinase; FBS, fetal bovine serum; GAP, GTPase-activating protein; GEF, guanine nucleotide-exchange factor; GST, glutathione S-transferase; HA, haemagglutinin; HEK-293, human embryonic kidney 293; LARG, leukaemia-associated RhoGEF; MAPK, mitogen-activated protein kinase; MBP, maltosebinding protein; Myr-B1, Src myristylation signal fused to the intracellular domain of plexin-B1; PAK, p21-activated kinase; PRG, PDZ-RhoGEF; RBD, Rho-GTP binding domain; Sema3A etc., semaphorin 3A etc.

1 To whom correspondence should be addressed, at Life Sciences Institute, University of Michigan, Ann Arbor, MI 48109, U.S.A. (email kunliang@umich.edu). 
Babco, antibodies against Myc and AU1 were from Covance, the antibody against FLAG was from Sigma, and the antibody against phospho-Raf $\left(\mathrm{Ser}^{338}\right)$ was from New England Biolabs. pcDNA3VSV-plexin-B1, pcDNA3-VSV-plexin-B1-HA, pRK5-myc-Rho, AU1, Sema3A-SEAP [AP (alkaline phosphatase)-Sema3A], Sema4D-SEAP (AP-Sema4D), FLAG-Raf, H-Ras constructs, HA-ERK and pRK5-GST-Sema4D have been described previously [12,20-22]. Myr-B1 [Src myristylation signal fused to the intracellular domain (amino acid residues 1612-1910) of plexinB1] was constructed in pcDNA3 plasmid. Myr-B1-FLAG was constructed from the plasmid containing Myr-B1 with the FLAG sequence inserted at the C-terminus. DN-LARG is amino acid residues 1-191 from LARG cloned into pCNF.

\section{Cell culture and kinase assay}

HT-22 cells and HEK-293 (human embryonic kidney 293) cells were maintained in DMEM (Dulbecco's modified Eagle's medium; Invitrogen) supplemented with $10 \%$ (v/v) FBS (fetal bovine serum) and $1.25 \mu \mathrm{g} / \mathrm{ml}$ penicillin/streptomycin. HEK-293 cells were transfected using Lipofectamine ${ }^{\circledR}$ (Invitrogen), according to the manufacturer's instructions, with the indicated plasmids. At $24 \mathrm{~h}$ post-transfection, cells were serum-starved in DMEM overnight. Cells were then harvested in RIPA buffer [50 mM Tris/HCl (pH 7.5), $150 \mathrm{mM} \mathrm{NaCl}, 1.0 \%$ Triton X-100, $0.5 \%$ deoxycholate, $0.1 \%$ SDS, $5 \mathrm{mM}$ EDTA, $25 \mathrm{mM} \mathrm{NaF}$ and $1 \mathrm{mM}$ DTT (dithiothreitol)]. HA-ERK was immunoprecipitated using the anti-HA antibody. Immunoprecipitated HA-ERK was then washed three times in RIPA buffer, once with Hepes buffer [25 mM Hepes (pH 7.5), 0.5 mM EDTA and $0.025 \%$ 2-mercaptoethanol] and once with kinase buffer [18 mM Hepes, ( $\mathrm{pH} 7.5)$, $10 \mathrm{mM}$ magnesium acetate, $10 \mu \mathrm{M}$ ATP and $1 \mathrm{mM}$ DTT]. Kinase buffer, $5 \mu \mathrm{g}$ of GST (glutathione S-transferase)-Elk and $2.5 \mu \mathrm{Ci}$ of $\left[\gamma-{ }^{32} \mathrm{P}\right]$ ATP were added to the immunoprecipitated HA-ERK. Reactions were incubated at $30^{\circ} \mathrm{C}$ for $30 \mathrm{~min}$. SDS-loading buffer [5 mM Tris/HCl (pH 6.8), $2 \%$ (w/v) SDS, $0.0125 \%$ Bromophenol Blue, $10 \%(\mathrm{w} / \mathrm{v})$ glycerol and $5 \%(\mathrm{v} / \mathrm{v}) 2$-mercaptoethanol] was added and samples were boiled. The samples were separated by SDS/PAGE and transferred on to a nitrocellulose membrane. The membrane was then exposed to a phosphoimager and the results were quantified.

\section{Generation of semaphorin proteins and AP staining}

$\mathrm{AP}$-semaphorin fusion proteins were expressed by transfection of HEK-293 cells with AP-Sema4D or AP-Sema3A expression constructs. Cells were grown in $5 \%(\mathrm{v} / \mathrm{v})$ FBS/DMEM and the medium was collected and concentrated. The medium was then dialysed overnight in $25 \mathrm{mM}$ Hepes buffer ( $\mathrm{pH}$ 8.0) prior to determination of the concentration by PNPP ( $p$-nitrophenyl phosphate) activity [23]. FPLC-purified Sema4D was purified by passing medium containing AP-Sema4D over a Q2 column (BioRad Laboratories).

HT-22 cells were treated with $2 \mathrm{nM}$ AP-Sema4D for $1 \mathrm{~h}$. Cells were washed three times with Hanks balanced salt solution [20 mM Hepes ( $\mathrm{pH} 7$ ) and $0.5 \mathrm{mg} / \mathrm{ml} \mathrm{BSA} \mathrm{(Invitrogen)]} \mathrm{and} \mathrm{fixed}$ with $4 \%(\mathrm{v} / \mathrm{v})$ paraformaldehyde and $10 \%(\mathrm{w} / \mathrm{v})$ sucrose in PBS for $10 \mathrm{~min}$. Cells were placed at $65^{\circ} \mathrm{C}$ for $1 \mathrm{~h}$ to inactivate endogenous phosphatases. Cells were washed three times with TMNT buffer [100 mM Tris/ $\mathrm{HCl}$ (pH 9.5), $100 \mathrm{mM} \mathrm{NaCl}, 50 \mathrm{mM}$ $\mathrm{MgCl}_{2}$ and $0.1 \%$ Tween-20]. The cells were then incubated with TMNT buffer containing $0.17 \mathrm{mg} / \mathrm{ml}$ BCIP (5-bromo-4chloroindol-3-yl phosphate) and $0.33 \mathrm{mg} / \mathrm{ml} \mathrm{NBT}$ (Nitro Blue Tetrazolium). Photomicrographs of the stained cells were taken with a Leica DM IRB light microscope after $24 \mathrm{~h}$.
pMT2-Sema4D and the control vector were transfected into HEK-293 cells, separately, by using Lipofectamine ${ }^{\circledR}$ (Invitrogen) [24]. At $48 \mathrm{~h}$ post-transfection, the medium was collected, concentrated and then dialysed in PBS overnight. Subsequently, $1 \%$ Triton X-100, $0.5 \mathrm{mM}$ EDTA, $0.1 \%$ 2-mercaptoethanol and glutathione-Sepharose ( $2 \mathrm{ml}$ of a $50 \%$ slurry) were added to the medium overnight. The beads were then washed three times with PBST (PBS containing $1 \%$ Triton X-100), once with $100 \mathrm{mM}$ Hepes (pH 8.0), and then GST-Sema4D was eluted in $100 \mathrm{mM}$ Hepes $(\mathrm{pH} 8.0)$ and $50 \mathrm{mM}$ glutathione. GSTSema4D was dialysed overnight in $25 \mathrm{mM}$ Hepes ( $\mathrm{pH} \mathrm{8.0)}$ ). Samples were then stained with Commassie Blue to determine the concentration.

\section{Generation of antibodies against plexin-B1, -A3 and -C1}

The intracellular domains of plexin-B1, -A3 and -C1 were expressed as MBP (maltose-binding protein) fusion proteins and purified. Two rabbits were immunized with purified MBP-plexinB1, MBP-plexin-A3 or MBP-plexin-C1 at the Pocono Rabbit Farm and Laboratory (Canadensis, PA, U.S.A.) and the antibodies produced.

\section{Rho activation assay}

HEK-293 cells were transfected with the indicated plasmids for $24 \mathrm{~h}$. Cells were then incubated in DMEM for $3 \mathrm{~h}$. Cells were lysed in RIPA buffer and cleared by centrifugation. Following centrifugation, $10 \mathrm{mM} \mathrm{MgCl}$ and $20 \mu \mathrm{g}$ of GST-Rhotekin-RBD (Rho-GTP binding domain) precoupled to glutathione-agarose beads were added to the soluble fraction for $45 \mathrm{~min}$. Beads were then washed with buffer [50 mM Tris/HCl (pH 7.5), $150 \mathrm{mM}$ $\mathrm{NaCl}$ and $1 \%$ Triton X-100]. Proteins were eluted with SDSloading buffer and resolved by SDS/PAGE. Myc-RhoA in the lysates and the pull-down assay was detected by Western blot using an anti-Myc antibody.

\section{RESULTS}

\section{Expression of plexin-B1 in HT-22 cells}

To investigate Sema4D signalling we wanted to first identify a cell type expressing plexin-B1. As previous studies have shown that plexin-B1 is expressed in the hippocampus [14], we therefore determined whether a mouse hippocampal cell line, HT-22, expressed plexin-B1. HT-22 cells were incubated with mocktransfected medium, AP-Sema3A or AP-Sema4D. The cells were then stained for AP activity. We found that HT-22 cells stained for the presence of AP-Sema4D; however, no staining occurred on cells that were treated with AP-Sema3A or mock-transfected medium (Figure 1A). These results indicate that HT-22 cells express a Sema4D receptor, but not a Sema3A receptor. To confirm that the plexin-B1 receptor was present in HT-22 cells, we performed a Western blot with an antibody against plexin-B1 (Figure 1B). The plexin-B1 antibody used was prepared in rabbits against recombinant $\mathrm{MBP}$-plexin-B1 protein and only recognizes plexin-B1 and not plexin-A3 or - $\mathrm{C} 1$ (Figure 1B, lower panel). HT-22 cells were found to contain plexin-B1, whereas COS-7 and HEK-293 cells did not (Figure 1B). This analysis indicates that plexin-B1 is expressed in HT-22 cells and is able to bind to AP-Sema4D.

\section{Phosphorylation of ERK in response to Sema4D treatment}

Netrin and Sema3A are axon guidance cues that result in activation of the MAPK pathway. Phosphorylation and activation of ERK in 
A

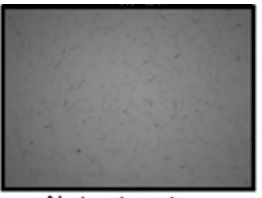

No treatment
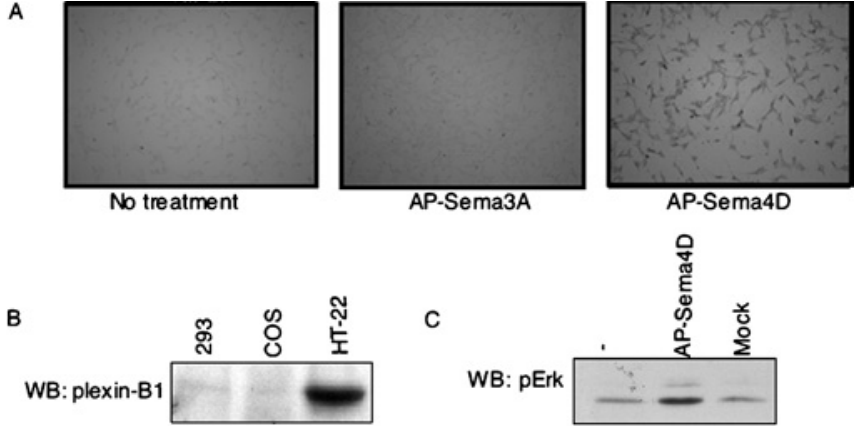

HA- A3 B1 C1

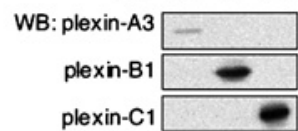

D

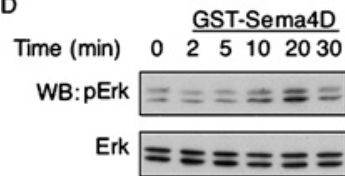

C

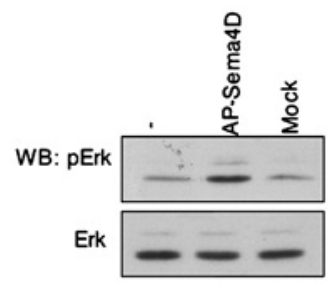

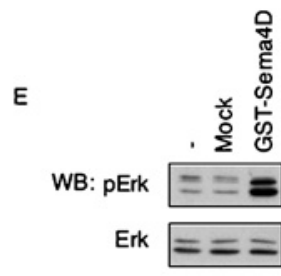

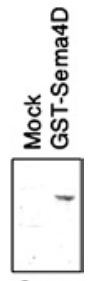

Comm
Figure 1 Phosphorylation of ERK in response to Sema4D treatment in HT-22 cells

(A) AP staining of HT-22 cells, a mouse hippocampal cell line. Cells were treated with specified proteins for $1 \mathrm{~h}$ and then stained for AP activity. (B) Presence of plexin-B1 in cell lines. Upper panel, HEK-293 (293), COS-7 and HT-22 cell lysates were analysed by Western blotting (WB) with an antibody against plexin-B1. Lower panel, characterization of antibodies against plexin-A3, -B1 and -C1. (C) Sema4D stimulates ERK phosphorylation. HT-22 cells were treated with either mock-transfected (Mock) or AP-Sema4D for $20 \mathrm{~min}$. Cells were lysed and analysed by Western blotting (WB) with antibodies against phospho-ERK (pERK) and ERK. (D) Time course of ERK activation downstream of purified GST-Sema4D. HT-22 cells were treated with $4 \mathrm{nM}$ GST-Sema4D for the times indicated. Cellular lysates were then analysed by Western blotting (WB) with antibodies against phospho-ERK (pERK) and ERK. (E) GST-Sema4D activates ERK. HT-22 cells were treated with $4 \mathrm{nM} \mathrm{GST-Sema4D}$ or mock-purified medium (Mock) for 20 min. Left-hand panel, cellular lysates were then analysed by Western blotting (WB) with antibodies against phospho-ERK (pERK) and ERK. Right-hand panel, Coomassie-Blue (Comm) stained GST-Sema4D and mock-purified medium (Mock).

response to these ligands contributes to axon turning and protein synthesis [16]. To determine whether ERK phosphorylation occurred downstream of Sema4D, HT-22 cells were treated with AP-Sema4D or purified mock-transfected medium. We observed that ERK phosphorylation was increased in response to APSema4D treatment (Figure 1C). To confirm further that ERK activation was a result of Sema4D stimulation, GST-Sema4D was expressed in HEK-293 cells, purified by a glutathione affinity column (Figure 1E, right-hand panel) and was used to determine the time course of ERK activation in HT-22 cells. ERK phosphorylation was first observed at $10 \mathrm{~min}$ and peaked at $20 \mathrm{~min}$ (Figures 1D and 1E). These data show the Sema4D treatment of HT-22 cells increases ERK phosphorylation.

\section{Requirement of the C-terminus of plexin-B1 for ERK activation}

In order to determine the mechanism of activation of ERK by Sema4D/plexin-B1, an overexpression system was employed. HEK-293 cells were transfected with plexin-B1 and were then treated with AP-Sema4D. The activity of co-transfected HAERK was analysed by performing an ERK kinase assay using Elk1 as a substrate. Cells expressing plexin-B1 had an increase in ERK activity towards recombinant GST-Elk1 in response to

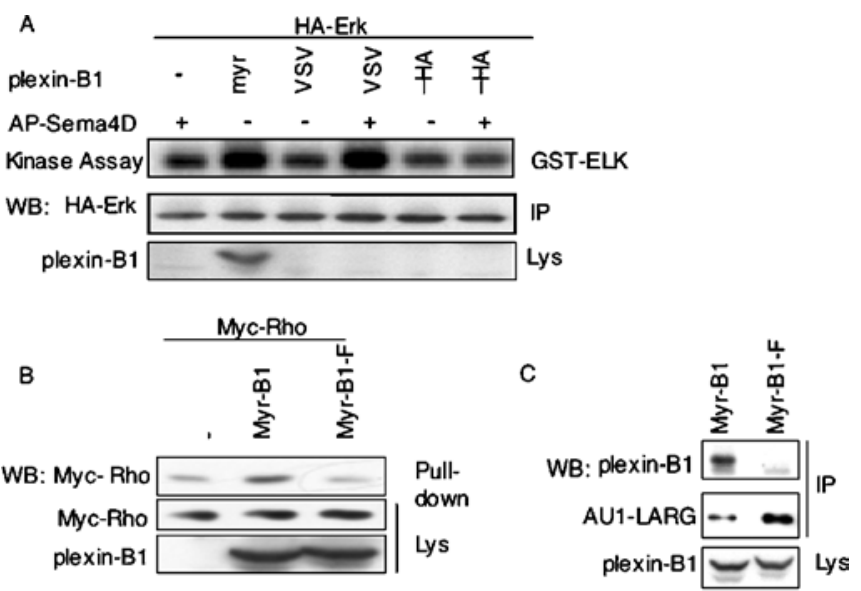

D

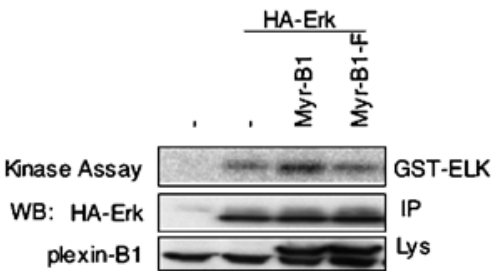

Figure 2 Requirement of the C-terminus of plexin-B1 for ERK activation

(A) ERK is activated downstream of plexin-B1 in HEK-293 cells. HEK-293 cells were transfected with the indicated plasmids and then treated without $(-)$ or with $(+)$ AP-Sema4D. ERK kinase assay was then performed using GST-Elk as a substrate. VSV, full-length plexin-B1; -HA, C-terminally tagged full-length plexin-B1; myr, Myr-B1. Note that the level of full-length plexin-B1 transfected was below the sensitivity of the Western blot. (B) RhoA activation by Myr-B1. Rhotekin pull-down assay was performed from HEK-293 cells transfected with the indicated plasmids. Expression of Myc-Rho and Myc-B1 were determined by Western blotting (WB) of the cell lysate (Lys). Myr-B1-F, FLAG epitope tag at the C-terminus of Myr-B1. (C) AU1-LARG Co-immunoprecipitates with Myr-B1. The indicated plasmids were transfected into HEK-293 cells, AU1-LARG was immunoprecipitated and a Western blot (WB) was performed with the indicated antibodies. (D) ERK activation by Myr-B1. ERK kinase assay was performed from HEK-293 cells transfected with indicated plasmids. IP, immunoprecipitation; Lys, whole-cell lysate.

AP-Sema4D treatment, unlike those left untreated (Figure 2A, lanes 3 and 4).

We [25] and others [14,26,27] have shown previously that the $\mathrm{C}$-terminus of plexin-B1 is necessary for downstream signalling. In order to determine whether the C-terminus of plexin-B1 was necessary in the activation of ERK, the C-terminus of plexinB1 was masked by adding an HA epitope tag. Cells expressing VSV-plexin-B1-HA and treated with AP-Sema4D did not exhibit increased ERK activity (Figure 2A, lanes 5 and 6), suggesting that Sema4D-induced ERK activity requires the C-terminus of plexin-B1.

\section{Activation of ERK by membrane-targeted plexin-B1}

To confirm further that ERK was activated in response to plexin$\mathrm{B} 1$ activation, a plasma-membrane-targeted form of plexin-B1 was created. The intracellular domain of plexin-B1 was fused to the Src myristylation signal (Myr-B1) that targets the fused intracellular domain to the plasma membrane. It has been shown [9] that deletion of the ecto-domain increases plexin-B1 signalling activity. To demonstrate that Myr-B1 was constitutively active, its ability to activate RhoA was analysed in a rhotekin pull-down assay. The Rho-binding domain of rhotekin specifically binds Rho-GTP and is a measure of Rho activation. Expression of $\mathrm{Myr}-\mathrm{B} 1$ resulted in an increase in the activation of RhoA, whereas 

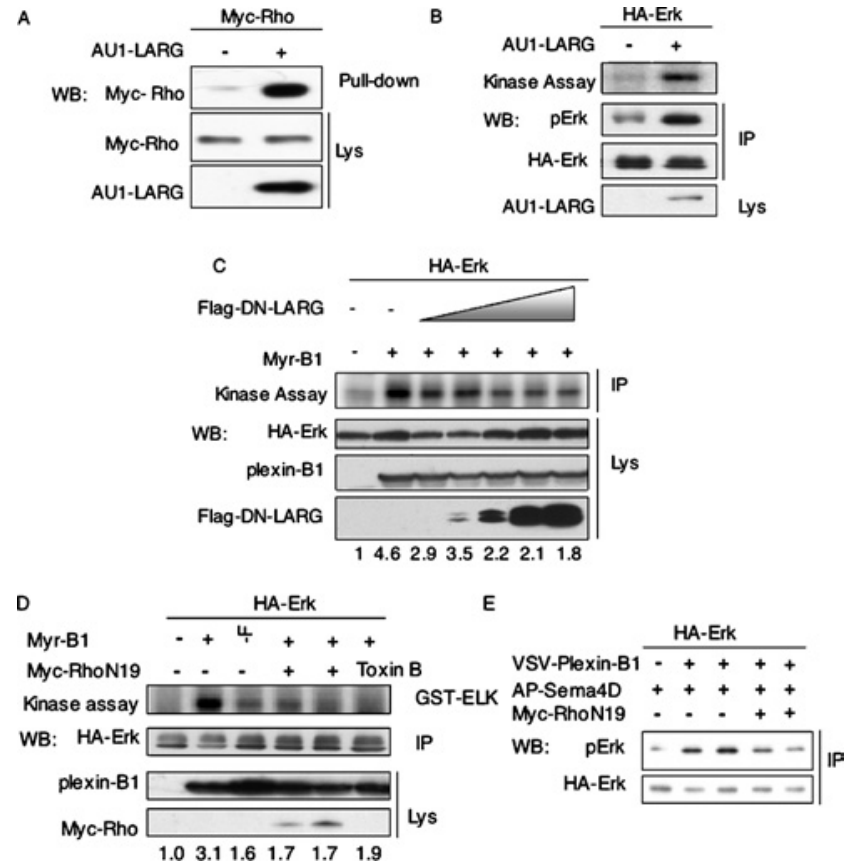

Figure 3 Contribution of activated Rho to ERK activation

(A) Overexpression of AU1-LARG activates RhoA. A rhotekin pull-down assay was performed from HEK-293 cells transfected with the indicated plasmids. (B) ERK activation through activation of RhoA. ERK kinase assay was performed from HEK-293 cells transfected with indicated plasmids. (C) Dominant-negative LARG blocks plexin-B1 activation of ERK. ERK kinase assay was performed from HEK-293 cells transfected with Myr-B1 and increasing concentrations of the PDZ domain of LARG (Flag-DN-LARG). (D) Dominant-negative RhoA and ToxinB inhibit ERK activation. ERK kinase assay was performed from HEK-293 cells transfected with indicated plasmids. Cells were treated with ToxinB for 30 min before harvesting. (E) Dominant-negative RhoA inhibits ERK activation downstream of Sema4D/plexin-B1. HEK-293 cells were transfected with the indicated plasmids. HA-ERK was immunoprecipitated (IP) and a Western blot was performed with the indicated antibodies. Note that the level of full-length plexin-B1 transfected was below the sensitivity of the Western blot. Lys, whole-cell lysates; WB, Western blot. In panels (C) and (D), the numbers indicate the activation of ERK relative to control (arbitrary units).

C-terminally blocked Myr-B1 did not (Figure 2B), corresponding to the ability of Myr-B1 to bind to LARG (Figure 2C). Furthermore, we observed an increase in COS-7 cell collapse in the presence of Myr-B1 (results not shown). In addition to activation of Rho, we observed that expression of Myr-B1 alone activated ERK in the absence of Sema4D (Figure 2A, lane 2 and Figure 2D). However, C-terminal-tagged Myr-B1 did not result in activation of ERK. These results are consistent with our observation (Figure 2A, lanes 5 and 6) that the C-terminal sequence of plexin-B1 is required for RhoA and ERK activation.

\section{Contribution of activated Rho to ERK activation}

We have shown previously [22] that GTP-bound Rho can signal to Raf to activate the MAPK cascade and ERK. The Rho exchange factor, LARG, binds to the C-terminus of plexin-B1 and activates RhoA upon Sema4D stimulation. Our hypothesis is that Sema4D activates ERK through plexin-B1 stimulation of LARG. This results in RhoA activation, which co-operates with Raf to signal downstream to MEK (MAPK/ERK kinase) and ERK. To test this hypothesis, we began by overexpressing LARG, which resulted in the activation of RhoA (Figure 3A). Subsequently, we tested whether overexpression of LARG increased ERK activity. We observed that LARG-mediated activation of RhoA resulted in enhanced ERK activity towards Elk (Figure 3B). We hypothesized that activation of RhoA by LARG may activate ERK.
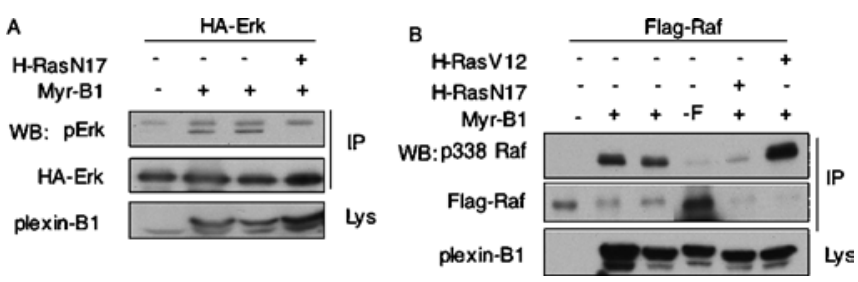

Figure 4 Co-operation of Rho with Ras to activate the MAPK pathway

(A) Dominant-negative H-Ras inhibits activation of ERK downstream of plexin-B1. HEK-293 cells were transfected with the indicated plasmids. HA-ERK was immunoprecipitated (IP) and a Western blot (WB) was performed with the indicated antibodies. (B) Raf is activated downstream of Myr-B1. FLAG-C-Raf was co-transfected into HEK-293 cells with the indicated plasmids. FLAG-C-Rat was immunoprecipitated (IP) and a Western blot (WB) was performed with the indicated antibodies. -F, FLAG tag at the C-terminus of the protein.

\section{Characterization of ERK activation downstream of plexin-B1}

To determine whether binding of LARG to the C-terminus of plexin-B1 was responsible for the activation of ERK, a portion of LARG containing only the PDZ domain, which acts as a dominant-negative (FLAG-DN-LARG), was transfected into HEK293 cells. We observed that dominant-negative LARG blocked the binding of endogenous LARG to Myr-B1 (results not shown) and caused a decreased ERK activation in response to Myr-B1 (Figure 3C). These data indicate that the C-terminus of plexin$\mathrm{B} 1$ and perhaps the binding of LARG are essential for the activation of ERK downstream of plexin-B1.

To ensure that activation of RhoA was responsible for activation of ERK downstream of plexin-B1, dominant-negative RhoA (RhoAN19) was co-transfected with Myr-B1 into HEK-293 cells and the activity of ERK was assayed. RhoAN19 expression blocked the plexin-B1-induced ERK activation (Figure 3D). To implicate RhoA activation further in the activation of ERK, cells were treated with ToxinB, which inhibits activation of Rho family GTPases. As predicted, cells expressing Myr-B1 had decreased ERK activity when treated with ToxinB (Figure 3D). In addition, ERK phosphorylation downstream of AP-Sema4D in plexinB1-expressing HEK-293 cells was inhibited by the expression of myc-RhoN19 (Figure 3E).

\section{Co-operation of Rho with Ras to activate the MAPK pathway}

Thus far we have shown that plexin-B1 activates the MAPK pathway and we have outlined the role of Rho in the activation of ERK. We have shown previously [22] that Rho co-operates with Ras to activate Raf in the MAPK pathway. To test this, we cotransfected Myr-B1 with a mutant of Ras that displays decreased activity (H-RasN17). When Myr-B1 was co-transfected with ERK, phosphorylation of ERK was increased. However, when H-Ras N17 was co-transfected, the phosphorylation of ERK was decreased (Figure 4A). This result suggested that plexin-B1 activation of the MAPK pathway required Ras. However, when Ras was co-transfected with Myr-B1, Ras GTP levels were not increased (results not shown). Therefore Ras may co-operate with Rho to activate the MAPK pathway.

To determine whether Raf was activated in the downstream signalling mechanisms of plexin-B1, the phosphorylation of Raf on $\operatorname{Ser}^{338}$ was investigated, the phosphorylation of which is required for Raf activity [22]. When Myr-B1 was co-transfected with FLAG-C-Raf, phosphorylation of $\operatorname{Ser}^{338}$ of Raf was increased (Figure 4B). Furthermore, when H-RasN17 was coexpressed, phosphorylation of Raf was decreased. Together, these data suggest that Ras co-operates with Rho to activate Raf. 


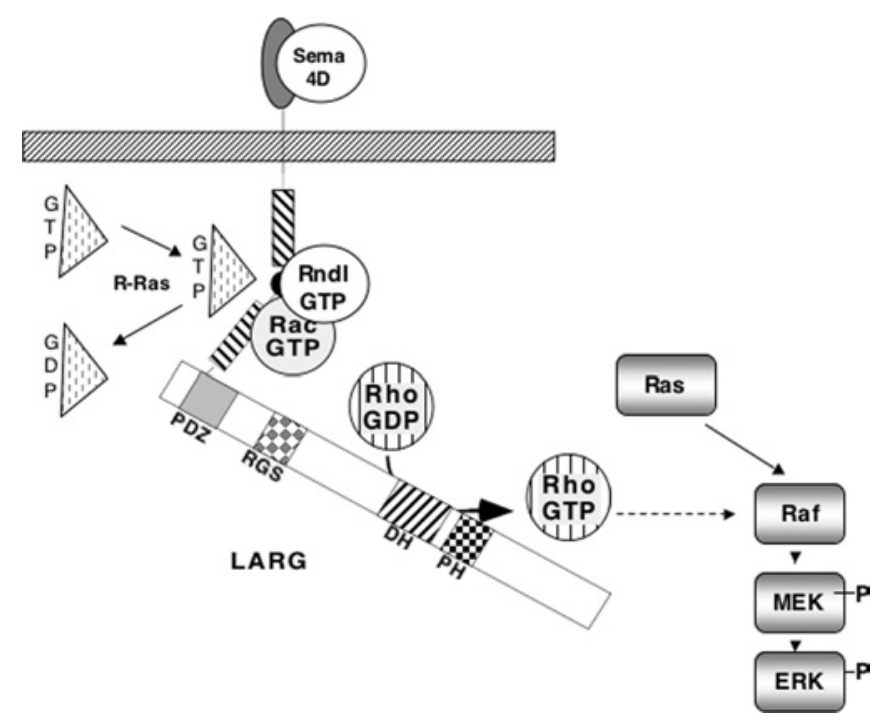

Figure 5 Potential pathway of plexin-B1 signalling

This model represents the known signalling pathways in addition to results in the present paper. $\mathrm{DH}$ and $\mathrm{PH}, \mathrm{Dbl}$ homology and pleckstrin homology domains respectively. MEK, MAPK/ERK kinase.

\section{DISCUSSION}

Semaphorins were initially discovered as axon guidance cues; however, they have also been shown to play a role in many different cellular types and developmental processes. Semaphorins participate in branching of the vasculature and lung and cellular migration within the immune system and cancer. The pathways that control the intracellular signalling of semaphorins regulate cellular structure, attachment and proliferation. Elucidation of signalling pathways thus far has shown that semaphorins modulate the cytoskeletal structure by regulation of Rho family of proteins and signal to cytoskeletal attachment by signalling through R-Ras. However, to date, the signalling pathways that control proliferation have yet to be analysed downstream of plexin-B family members.

Activation of the MAPK pathways has been implicated as signalling events downstream of many axon guidance proteins. The role of MAPK activation downstream of semaphorin signalling may play a role in controlling the cytoskeleton [16]. Moreover, MAPK activation has also been shown to cause an increase in protein synthesis in the growth cone downstream of Netrin and Sema3A [16]. In addition, ERK has been shown previously $[18,19]$ to regulate the outgrowth of neurites downstream of netrin treatment. Cell survival in neurons has been shown to be regulated by ERK playing an anti-apoptotic role; in contrast, sustained over-stimulation can promote necrotic neuronal cell death [28]. With the varying roles of MAPK activation in neuronal cells, further research is needed to understand the role of MAPK activation downstream of Sema4D.

In the present study, we have shown that ERK is activated downstream of plexin-B1. Incubation of Sema4D with a mouse hippocampal cell line increases the phosphorylation and activation of ERK. Furthermore, Sema4D activates ERK in transfected cells via signalling through the $\mathrm{C}$-terminus of plexin-B1. We [25] and others $[14,26,27]$ have shown previously that LARG, a GEF for the small GTPase RhoA, binds the C-terminus of plexin-B1 and is activated upon Sema4D stimulation. In the present study, we have shown that inhibition of LARG binding and activation of Rho inhibits plexin-B1 activation of ERK. Furthermore, we have shown that Rho co-operates with Ras to activate Raf. Based on the data described in the present study, we would like to propose that activation of RhoA stimulates the MAPK pathway downstream of plexin-B1 (Figure 5).

Weacknowledge DrH. G. Vikis for critical reading of the manuscript prior to submission. We thank T. Zhu for technical assistance. We also thank Dr D. Schubert (Cellular Neurobiology Laboratory, The Salk Institute for Biological Sciences, La Jolla, CA, U.S.A.) for the HT-22 cell line, Dr P. M. Comoglio (Division of Molecular Oncology, Institute for Cancer Research and Treatment, University of Torino Medical School, Torino, Italy) for the pMT2-Sema4D construct, and Dr J. S. Gutkind (Oral and Pharyngeal Cancer Branch, National Institute of Dental and Craniofacial Research, National Institutes of Health, Bethesda, MD, U.S.A.) for AU1-LARG. J. A. is supported by grant GM07767 from NIGMS (National Institute of General Medical Sciences). This work was supported by grants from the National Institutes of Health (to K. -L.G.).

\section{REFERENCES}

1 Spriggs, M. K. (1999) Shared resources between the neural and immune systems: semaphorins join the ranks. Curr. Opin. Immunol. 11, 387-391

2 Christensen, C. R., Klingelhofer, J., Tarabykina, S., Hulgaard, E. F., Kramerov, D. and Lukanidin, E. (1998) Transcription of a novel mouse semaphorin gene, M-semaH, correlates with the metastatic ability of mouse tumor cell lines. Cancer Res. $\mathbf{5 8}$, 1238-1244

3 Behar, O., Golden, J. A., Mashimo, H., Schoen, F. J. and Fishman, M. C. (1996) Semaphorin III is needed for normal patterning and growth of nerves, bones and heart. Nature (London) 383, 525-528

4 Basile, J. R., Barac, A., Zhu, T., Guan, K. L. and Gutkind, J. S. (2004) Class IV semaphorins promote angiogenesis by stimulating Rho-initiated pathways through plexin-B. Cancer Res. 64, 5212-5224

5 Ito, T., Kagoshima, M., Sasaki, Y., Li, C., Udaka, N., Kitsukawa, T., Fujisawa, H., Taniguchi, M., Yagi, T., Kitamura, H. and Goshima, Y. (2000) Repulsive axon guidance molecule Sema3A inhibits branching morphogenesis of fetal mouse lung. Mech. Dev. 97 $35-45$

6 Feiner, L., Webber, A. L., Brown, C. B., Lu, M. M., Jia, L., Feinstein, P., Mombaerts, P., Epstein, J. A. and Raper, J. A. (2001) Targeted disruption of semaphorin 3C leads to persistent truncus arteriosus and aortic arch interruption. Development 128, 3061-3070

7 Brown, C. B., Feiner, L., Lu, M. M., Li, J., Ma, X., Webber, A. L., Jia, L., Raper, J. A. and Epstein, J. A. (2001) PlexinA2 and semaphorin signaling during cardiac neural crest development. Development 128, 3071-3080

8 Oinuma, I., Katoh, H. and Negishi, M. (2004) Molecular dissection of the semaphorin 4D receptor plexin-B1-stimulated R-Ras GTPase-activating protein activity and neurite remodeling in hippocampal neurons. J. Neurosci. 24, 11473-11480

9 Zhang, Z., Vuori, K., Wang, H., Reed, J. C. and Ruoslahti, E. (1996) Integrin activation by R-ras. Cell 85, 61-69

10 Vikis, H. G., Li, W., He, Z. and Guan, K. L. (2002) The semaphorin receptor plexin-B1 specifically interacts with active Rac in a ligand-dependent manner. Proc. Natl. Acad. Sci. U.S.A. 97, 12457-12462

11 Driessens, M. H., Hu, H., Nobes, C. D., Self, A., Jordens, I., Goodman, C. S. and Hall, A. (2001) Plexin-B semaphorin receptors interact directly with active Rac and regulate the actin cytoskeleton by activating Rho. Curr. Biol. 11, 339-544

12 Vikis, H. G., Li, W. and Guan, K. L. (2002) The plexin-B1/Rac interaction inhibits PAK activation and enhances Sema4D ligand binding. Genes Dev. 16, 836-845

13 Hu, H., Marton, T. F. and Goodman, C. S. (2001) Plexin B mediates axon guidance in Drosophila by simultaneously inhibiting active Rac and enhancing RhoA signaling. Neuron 32, 39-51

14 Swiercz, J. M., Kuner, R., Behrens, J. and Offermanns, S. (2002) Plexin-B1 directly interacts with PDZ-RhoGEF/LARG to regulate RhoA and growth cone morphology. Neuron 35, 51-63

15 Pasterkamp, R. J., Peschon, J. J., Spriggs, M. K. and Kolodkin, A. L. (2003) Semaphorin $7 A$ promotes axon outgrowth through integrins and MAPKs. Nature (London) 424, 398-405

16 Campbell, D. S. and Holt, C. E. (2003) Apoptotic pathway and MAPKs differentially regulate chemotropic responses of retinal growth cones. Neuron 37, 939-952

17 Perron, J. C. and Bixby, J. L. (1999) Distinct neurite outgrowth signaling pathways converge on ERK activation. Mol. Cell. Neurosci. 13, 362-378

18 Forcet, C., Stein, E., Pays, L., Corset, V., Llambi, F., Tessier-Lavigne, M. and Mehlen, P. (2002) Netrin-1-mediated axon outgrowth requires deleted in colorectal cancerdependent MAPK activation. Nature (London) 417, 443-447

19 Ming, G. L., Wong, S. T., Henley, J., Yuan, X. B., Song, H. J., Spitzer, N. C. and Poo, M. M. (2002) Adaptation in the chemotactic guidance of nerve growth cones. Nature (London) 417, 411-418 
20 Tamagnone, L., Artigiani, S., Chen, H., He, Z., Ming, G. I., Song, H., Chedotal, A., Winberg, M. L., Goodman, C. S., Poo, M. et al. (1999) Plexins are a large family of receptors for transmembrane, secreted, and GPI-anchored semaphorins in vertebrates. Cell 99, 71-80

21 Fukuhara, S., Murga, C., Zohar, M., Igishi, T. and Gutkind, J. S. (1999) A novel PDZ domain containing guanine nucleotide exchange factor links heterotrimeric $G$ proteins to Rho. J. Biol. Chem. 274, 5868-5879

$22 \mathrm{Li}, \mathrm{W}$. , Chong, H. and Guan, K. L. (2001) Function of the Rho family GTPases in Ras-stimulated Raf activation. J. Biol. Chem. 276, 34728-34737

23 Flanagan, J. G. and Leder, P. (1990) The kit ligand: a cell surface molecule altered in steel mutant fibroblasts. Cell 63, 185-194

24 Giordano, S., Corso, S., Conrotto, P., Artigiani, S., Gilestro, G., Barberis, D., Tamagnone, L. and Comoglio, P. M. (2002) The semaphorin 4D receptor controls invasive growth by coupling with Met. Nat. Cell Biol. 4, 720-724

Received 12 July 2005/27 September 2005; accepted 28 September 2005

Published as BJ Immediate Publication 28 September 2005, doi:10.1042/BJ20051123
25 Aurandt, J., Vikis, H. G., Gutkind, J. S., Ahn, N. and Guan, K. L. (2002) The semaphorin receptor plexin-B1 signals through a direct interaction with the Rho-specific nucleotide exchange factor, LARG. Proc. Natl. Acad. Sci. U.S.A. 99, 12085-12090

26 Perrot, V., Vazquez-Prado, J. and Gutkind, J. S. (2002) Plexin B regulates Rho through the guanine nucleotide exchange factors leukemia-associated Rho GEF (LARG) and PDZ-RhoGEF. J. Biol. Chem. 277, 43115-43120

27 Hirotani, M., Ohoka, Y., Yamamoto, T., Nirasawa, H., Furuyama, T., Kogo, M., Matsuya, T. and Inagaki, S. (2002) Interaction of plexin-B1 with PDZ domain-containing Rho guanine nucleotide exchange factors. Biochem. Biophys. Res. Commun. 297, 32-37

28 Runden, E., Seglen, P. O., Haug, F. M., Ottersen, O. P., Wieloch, T., Shamloo, M. and Laake, J. H. (1998) Regional selective neuronal degeneration after protein phosphatase inhibition in hippocampal slice cultures: evidence for a MAP kinase-dependent mechanism. J. Neurosci. 18, 7296-7305 